\title{
Crossmodal Classification of Mu Rhythm Activity during Action Observation and Execution Suggests Specificity to Somatosensory Features of Actions
}

\author{
@Michel-Pierre Coll, ${ }^{1,2}$ Clare Press, ${ }^{3}{ }^{\oplus}$ Hannah Hobson, ${ }^{2}{ }^{\circledR C}$ Caroline Catmur, ${ }^{4}$ and Geoffrey Bird ${ }^{1,2}$ \\ ${ }^{1}$ Department of Experimental Psychology, University of Oxford, Oxford SE5 8AF, United Kingdom, ${ }^{2}$ MRC Social, Genetic and Developmental Psychiatry \\ Centre, Institute of Psychiatry, Psychology and Neuroscience, King's College London, University of London, London SE5 8AF, United Kingdom, \\ ${ }^{3}$ Department of Psychological Sciences, Birkbeck, University of London, London WC1E 7HX, United Kingdom, and ${ }^{4}$ Department of Psychology, Institute of \\ Psychiatry, Psychology and Neuroscience, King's College London, University of London, London SE5 8AF, United Kingdom
}

The alpha mu rhythm $(8-13 \mathrm{~Hz})$ has been considered to reflect mirror neuron activity because it is attenuated by both action observation and action execution. The putative link between mirror neuron system activity and the mu rhythm has been used to study the involvement of the mirror system in a wide range of socio-cognitive processes and clinical disorders. However, previous research has failed to convincingly demonstrate the specificity of the mu rhythm, meaning that it is unclear whether the mu rhythm reflects mirror neuron activity. It also remains unclear whether mu rhythm suppression during action observation reflects the processing of motor or tactile information. In an attempt to assess the validity of the mu rhythm as a measure of mirror neuron activity, we used crossmodal pattern classification to assess the specificity of EEG mu rhythm response to action varying in terms of action type (whole-hand or precision grip), concurrent tactile stimulation (stimulation or no stimulation), or object use (transitive or intransitive actions) in 20 human participants. The main results reveal that above-chance crossmodal classification of mu rhythm activity was obtained in the central channels for tactile stimulation and action transitivity but not for action type. Furthermore, traditional univariate analyses applied to the same data were insensitive to differences between conditions. By calling into question the relationship between mirror system activity and the mu rhythm, these results have important implications for the use and interpretation of mu rhythm activity.

Key words: action observation; EEG; mirror neurons; mu rhythm; multivariate pattern classification

Significance Statement

The central alpha mu rhythm oscillation is a widely used measure of the human mirror neuron system that has been used to make important claims concerning cognitive functioning in health and in disease. Here, we used a novel multivariate analytical approach to show that crossmodal EEG mu rhythm responses primarily index the somatosensory features of actions, suggesting that the mu rhythm is not a valid measure of mirror neuron activity. Results may lead to the revision of the conclusions of many previous studies using this measure, and to the transition toward a theory of mu rhythm function that is more consistent with current models of sensory processing in the self and in others.

\section{Introduction}

Mirror neurons (MNs), firing both during the observation and execution of actions (di Pellegrino et al., 1992), have been sug- gested to contribute to the understanding of others' action by matching observed actions to one's own neural code to perform that action (Gallese et al., 1996). MN activity is notoriously difficult to measure noninvasively in humans given the limited spatial resolution of human neuroimaging techniques (Dinstein et al., 2008). Despite these methodological constraints, many have argued for the involvement of MN in a variety of phenomena using neuroimaging measures, such as the EEG central alpha mu rhythm. 
The alpha mu rhythm $(8-13 \mathrm{~Hz}$, henceforth "mu rhythm") is an oscillation measured over sensorimotor areas that is attenuated both during the observation and execution of actions (Fox et al., 2016). On the basis of this similar response during action observation and execution, the mu rhythm has been considered to index MN activity (Pineda, 2005; Fox et al., 2016). As a consequence, it has been used by many researchers to suggest the involvement of $\mathrm{MN}$ throughout development in processes such as empathy (Gallese, 2001; Cheng et al., 2008; Yang et al., 2009), theory of mind (Pineda and Hecht, 2009), speech perception (Moreno et al., 2013), and many other socio-cognitive processes (for review, Vanderwert et al., 2013). Furthermore, differences in mu rhythm response between clinical and typical samples have been used to suggest atypical MN response in conditions, such as autism spectrum disorder (Oberman et al., 2005, 2008; Bernier et al., 2007, 2013), schizophrenia (Singh et al., 2011; McCormick et al., 2012; Horan et al., 2014), and addiction (Pineda and Oberman, 2006).

There are, however, two important issues with the claim that mu rhythm indexes MN activity. First, most studies do not provide a convincing demonstration of the specificity of mu rhythm response. For the mu rhythm to be considered a valid index of MN, it should show crossmodal action specificity: that is, the response associated with one action should be similar whether it is observed or executed (crossmodality), but it should also be distinguishable for different actions (specificity) (Kilner and Lemon, 2013; Oosterhof et al., 2013). Without demonstration of specificity, it is possible that the similarity of mu rhythm responses during action observation and execution reflects general effects of task engagement, attention, readiness to act, or arousalrelated activation involving nonmirror neuronal populations (Dinstein et al., 2008; Cook et al., 2014). Second, empirical evidence suggests that the mu rhythm might index sensory processing rather than motor activity (Cheyne et al., 2003; Ritter et al., 2009; Coll et al., 2015). Thus, the mu rhythm may index the observation and receipt of tactile stimulation rather than the observation and execution of actions.

Given that the mu rhythm is often used to assert the involvement of MN in a variety of cognitive processes and clinical conditions, it is important to ensure its validity as an index of MN activity by verifying that it shows crossmodal specificity to observed and executed actions. Accordingly, we used crossmodal pattern classification to assess the specificity of the mu rhythm during action observation and action execution. Furthermore, the degree of tactile stimulation during action observation and execution was manipulated both by the application of a vibratory tactile stimulation to the hand and by action transitivity (whether actions were, or were not, object-directed; the former, but not the latter, generating cutaneous tactile stimulation). If the mu rhythm represents $\mathrm{MN}$ activity, then crossmodal classification of two different types of actions should be accurate at above-chance levels, and superior to the classification of tactile stimulation. In contrast, if the mu rhythm indexes tactile mirroring, classification accuracy should be above chance when classifying the presence or absence of tactile stimulation, but at chance for action type.

\section{Materials and Methods}

\section{Experimental design}

The crossmodal specificity of the mu rhythm to two different actions (Action type factor) was assessed while manipulating the amount of tactile stimulation involved in these actions in two different ways. The first manipulation related to the presence or absence of an external tactile stimulation (Vibration factor), and the second concerned whether the actions were directed toward an object or simply mimed (Transitivity factor). To assess the crossmodal specificity of the mu rhythm to action or stimulation type, and to maximize the number of trials that could be used for multivariate classification, we analyzed the data using a fractional factorial design in which only the main effect of each condition was investigated. We therefore independently tested the effect of Action type, Vibration, and Transitivity while collapsing across the two other conditions. If the mu rhythm is specific to the action observed and executed, then the crossmodal classifier should be able to discriminate the two actions. If the mu rhythm is sensitive to differences in tactile stimulation, then the classifier should be able to discriminate between the presence and absence of the vibration and between transitive and intransitive actions. We predicted that crossmodal classification accuracy in central channels would increase with the strength of the difference in tactile stimulation in each condition and would thus follow a Vibration $>$ Transitivity $>$ Action type pattern. We used three main approaches to test this crossmodal classification. First, to investigate the scalp distribution of the effects, we performed exploratory classification analyses on the time-frequency activity of the mu rhythm on each channel and its neighbors using a spatial searchlight approach (Kriegeskorte et al., 2006). Second, to visualize the neural sources contributing to the observed scalp effects, a spatial searchlight was also used on mu rhythm activity at the source level. Finally, to test the claim that the central mu rhythm shows crossmodal specificity, region of interest (ROI) analyses were performed using a crossmodal classifier in a central cluster of channels selected according to the mu rhythm literature. To ensure that any crossmodal effect observed in this central cluster is specific to the central alpha mu rhythm and not confounded with the occipital alpha rhythm (Hobson and Bishop, 2016), these analyses were performed at both central and occipital scalp locations. We predicted that crossmodal classification would be observed only at the central location.

\section{Participants}

Twenty healthy right-handed adults ( 12 females) aged on average 24.60 years $(\mathrm{SD}=6.75$, range $=19-49$ years $)$ were recruited through university-wide advertisements and gave written informed consent to take part in this study. Exclusion criteria included being over 50 years old or any reported history of neurological or psychiatric disorder. The study was approved by King's College London Psychiatry, Nursing and Midwifery Research Ethics Subcommittee, and participants received an honorarium for their participation.

\section{EEG recordings}

EEG activity was acquired from a 61 channel (extended 10-20 montage) DC-coupled recording system (Brain Products, RRID:SCR_009443). Three additional EOG electrodes were placed below the left eye and at $1 \mathrm{~cm}$ from the outer canthi. The sampling rate was $500 \mathrm{~Hz}$, with reference at $\mathrm{FCz}$ and ground at $\mathrm{AFz}$. Impedances were maintained $<10 \mathrm{k} \Omega$.

\section{Vibration stimulator}

A custom-built stimulator was fixed on the back of the participant's right hand using medical tape. This stimulator consisted of two round cell phone micro vibration motors $(10 \times 2.7 \mathrm{~mm})$ vibrating at $\sim 10,000$ rotations per minute placed side by side on a piece of thin cardboard and sealed with black electrical tape. When the stimulator was turned on, it produced a continuous vibrating sensation on the back of the hand. A yellow LED light was placed on the top of the motors and was lit when the stimulator was turned on. The stimulator was wired into a USB relay switch and controlled by the stimuli presentation software (E-prime 2.0, Psychology Software Tools, RRID:SCR:009567). The wire was fixed onto the participant's forearm with medical tape to ensure that it did not interfere with action execution during the experimental task. A second identical stimulator was placed near the participant's arm and turned on during trials in which the hand stimulator remained off to create a similar sound.

\section{Visual stimuli}

The visual stimuli consisted of 3000 ms video clips depicting a hand wearing the vibration stimulator executing one of the 6 types of actions 
varying according to Action type (Precision grip, Whole-hand grip), Vibration (Vibration on, Vibration off) and Transitivity (Transitive, Intransitive) filmed from a first-person point of view (Fig. 1). During the Transitive trials, the hand was seen executing one of two actions on an empty plastic bottle placed on a black table. For the Intransitive trials, the bottle was absent from the screen and the same actions were mimed without the bottle. For Precision grip trials, the hand started flat on the table at the right of the screen, picked up (or mimed picking up) the bottle using a thumb and index grip on the cap of the bottle, and raised it $\sim 15 \mathrm{~cm}$ before placing it back on the table. For the Whole-hand grip trials, the hand picked up (or mimed picking up) the bottle using a wholehand grip on the body of the bottle. During Vibration on trials, the stimulator was turned on, and this was visible due to the vibration of the stimulator and the yellow LED light. During Vibration off actions, the stimulator was not turned on. All video clips were presented without sound. Two models (one female) were recorded while executing the actions to the beats of a metronome to ensure similar timing during all video clips. The models executed the actions twice for a total of 32 different stimuli ( 8 types $\times 2$ models $\times 2$ executions). The video clips were presented on a 17 -inch monitor located at $\sim 60 \mathrm{~cm}$ from the participant using the E-Prime 2.0 software (Psychology Software Tools, RRID:SCR:009567).

\section{Procedure}

Participants sat in a dimly lit room. After giving informed consent, the EEG cap and the stimulator were installed and participants received verbatim instructions for the task. During the experimental task, participants were asked to either observe the video clips or to execute one of the six action types using the same plastic bottle as in the video clips. To ensure that the bottle did not fall during the experiment, it was stabilized using a square piece of cardboard fixed at its base. A practice session was performed during which each of the six action types was first observed in a video clip and then executed by the participants using the plastic bottle. During this practice session, participants experienced the vibro-tactile stimulation and observed the lighting of the LED. They were explicitly instructed that the hand in the video clips wore the same vibration stimulator, and that this hand received the same vibrating stimulation when the LED light was turned on. The practice session was repeated if necessary to ensure that all participants understood the instructions and executed the actions in a correct manner with appropriate timing.

After the practice session, an occlusion box was placed over the participant's arm to prevent the participant from observing his or her actions and the LED light during the experiment. Movements were monitored using a webcam placed inside this box, and trials with incorrect action execution or with movement during observation were noted and removed from the analyses. All experimental conditions were blocked within mini-blocks of 10 trials during which participants either executed or observed the same action type 10 times. During Execution blocks, participants first saw the instructions indicating which action type should be executed (e.g., "Execute, Fine $O R$ Full grip, With $O R$ Without the object, With OR Without vibration) for $5000 \mathrm{~ms}$ followed by 10 trials consisting of an $800 \mathrm{~ms}$ green fixation cross, a $1000-5000 \mathrm{~ms}$ jittered white fixation cross, and a $3000 \mathrm{~ms}$ green circle. Participants were instructed to blink during the instructions and the green fixation cross but to refrain from blinking for the rest of the task. Participants were told to begin executing the action as soon as they saw the green circle and to have their hand back on the table before the green circle disappeared. During Vibration on trials, the vibration stimulator was turned on during the presentation of the green circle. During Vibration off trials, a second stimulator was turned on to produce a similar sound.
During Observation blocks, participants received the instruction "Please remain still and watch the video clips" followed by 10 trials consisting of the green and white fixation crosses presented for the same duration as the Execution blocks and a video clip. The stimulator was never turned on during the Observation blocks. Eight of the 28 Observation blocks were catch blocks during which one of the 10 video clips was presented with a red dot in the center. At the end of all Observation blocks, participants saw a prompt asking them to indicate whether they saw a red dot in one of the video clips using their left hand placed on a keyboard. Catch blocks were not included in the EEG analyses. Finally, during Baseline blocks, participants were instructed to remain still and to wait for the next instruction and then observed a black screen for $21 \mathrm{~s}$.

Participants performed 32 execution or observation blocks of 10 trials, two for each of the eight experimental condition [Vibration (On, Off) $\times$ Transitivity (Object, No object) $\times$ Action type (Precision, Wholehand)]. This resulted in 160 observation and 160 execution trials that were used for analyses. Eight catch trial blocks were used to ensure continuous attention to the stimuli and were not included in the EEG analyses. Participants performed four experimental sessions in which four execution, four observation, and two catch blocks were presented in a random order. Three baseline blocks were presented at the beginning, middle, and end of the session. The duration of the task was $\sim 60 \mathrm{~min}$, and participants were encouraged to take breaks between each session.

\section{Statistical analyses}

All EEG analyses were performed with the FieldTrip (Oostenveld et al., 2011, RRID:SCR_004849) and CosMoMVPA (Oosterhof et al., 2016, RRID:SCR_014519) toolboxes within MATLAB 2016a (The MathWorks, RRID:SCR_001622). The analyses workflow for both univariate and multivariate analyses is detailed in Figure 2. While the crossmodal specificity of the alpha band $(8-13 \mathrm{~Hz})$ was the main focus of this study, all analyses were also performed in the beta band (15-25 Hz) for completeness and in line with previous suggestions of a link between beta rhythm suppression and MN activity (e.g., Rossi et al., 2002).

\section{Catch trial accuracy}

Responses to the prompts presented after each observation blocks were scored as 0 (miss or false alarm) or 1 (correct detection or correct rejection) and averaged to obtain a catch trial accuracy score for each participant.

\section{EEG preprocessing}

EEG data were first bandpass filtered between 1 and $50 \mathrm{~Hz}$, and an additional $50 \mathrm{~Hz}$ notch filter was used to reduce electrical noise. The data were then epoched -1000 to $3500 \mathrm{~ms}$ relative to the onset of the video clips or the execution cue. Epochs of the same length were also taken from the baseline periods. This led to a total of 160 observation and 160 execution 
Analysis workflow

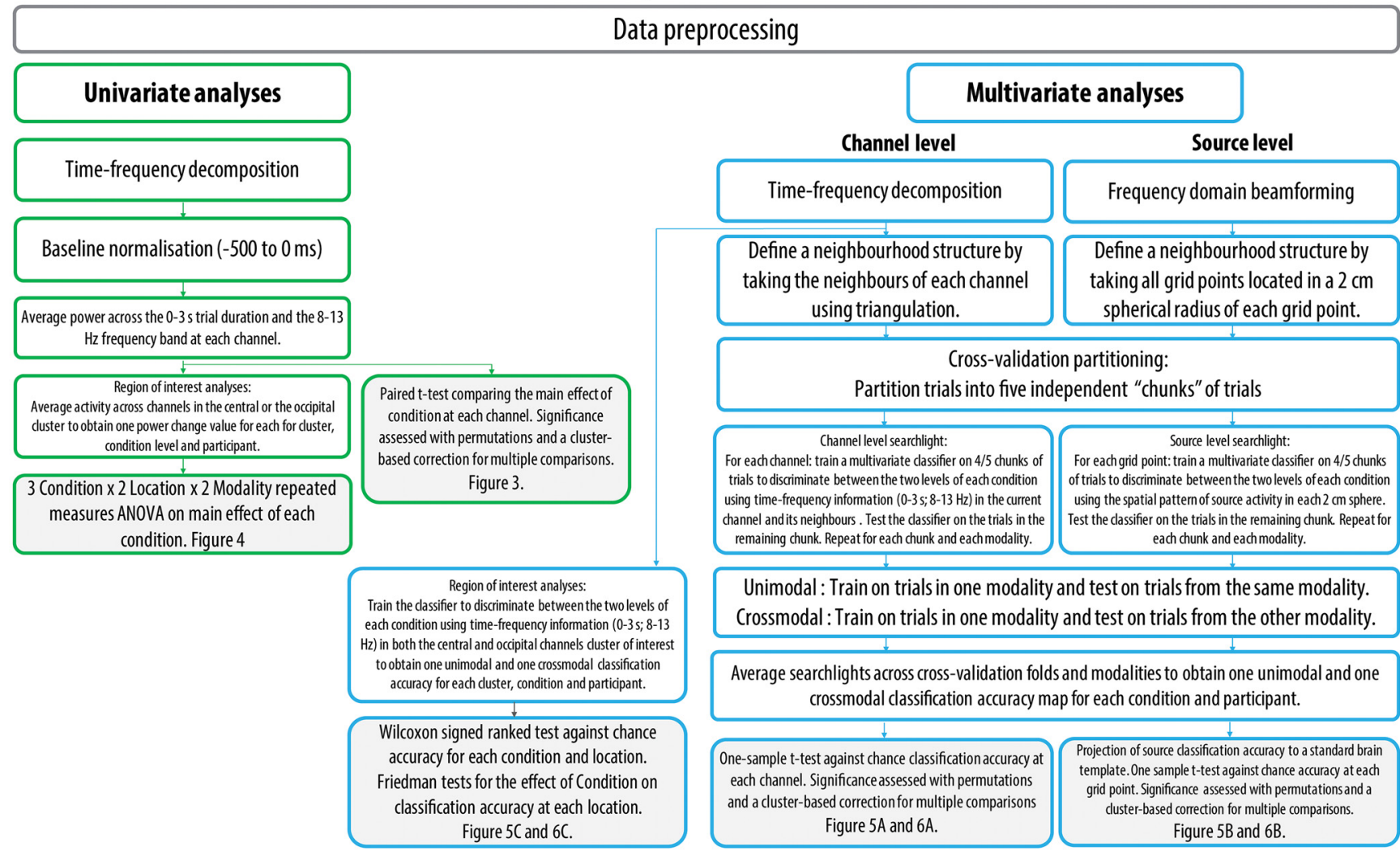

Figure 2. Schematic illustration of the analysis workflow for the univariate analyses (green borders) and the multivariate analyses (blue borders) performed at the channel and source levels. White boxes represent analyses performed at the subject level. Boxes with gray shading represent analyses performed at the group level.

trials. Independent component analyses were used to remove from the signal components that were associated with eye blinks, movements, or other obvious artifacts. The data were then visually inspected, and channels that were consistently bad throughout the experiment or trials with artifacts were removed from the analyses. Additionally, trials for which the participant performed the incorrect action or moved when they were not supposed to move were removed from the analyses. These procedures led to the removal of an average of $4.81 \%(\mathrm{SD}=3.68$, range $=$ $2 \%-15 \%)$ of trials. After epoch rejection, removed channels were interpolated using the average activity of neighboring channels.

For analyses at the channel level, the time-frequency representation of the data was obtained by applying a Fourier transformation in Hanningtapered sliding time windows with a fixed length of $500 \mathrm{~ms}$ and moving in steps of $50 \mathrm{~ms}$. Power was calculated from 5 to $30 \mathrm{~Hz}$ in steps of $1 \mathrm{~Hz}$.

For analyses at the source level, sources were identified using Dynamic Imaging of Coherent Sources (Gross et al., 2001), a frequency domain beamforming technique. Activity was source localized in a $250-2750 \mathrm{~ms}$ time window. A frequency of $10 \mathrm{~Hz}$ with a smoothing window of $\pm 2 \mathrm{~Hz}$ was used for the alpha band, and a frequency of $20 \mathrm{~Hz}$ with a smoothing window of $\pm 5 \mathrm{~Hz}$ was used for the beta band. These time and frequency windows were selected following visual inspection of the univariate effects on the basis of those time and frequency windows, which included the majority of the alpha and beta suppression. In brief, a volume conductor model was built for all participants using the boundary element method and a standard MNI template. A 10-mm-spaced dipole grid was wrapped onto the MNI brain template, and a normalized lead field was calculated. Dynamic Imaging of Coherent Sources was performed for each trial using a common spatial filter computed from the combination of all trials and a $5 \% \lambda$ regularization parameter. This resulted in the estimation of the alpha activity at each grid point for each participant and trial.

\section{EEG analyses}

Univariate analyses. To compare the mu rhythm suppression in the current experiment to that obtained in previous studies, we first analyzed our data using traditional univariate analyses. For these analyses, the average power in each condition was normalized relative to a -500 to 0 $\mathrm{ms}$ prestimulus baseline. Power was then averaged across frequency (alpha: $8-13 \mathrm{~Hz}$; beta: $15-25 \mathrm{~Hz}$ ) and time $(0-3000 \mathrm{~ms})$.

Exploratory analyses were first performed to investigate the scalp distribution of the main effect of each condition within each modality. To this end, a two-tailed paired sample $t$ test comparing the two levels of each condition was performed at each channel. The significance of this test was assessed using a nonparametric permutation approach in which this test was performed 10,000 times on the same data with randomly permuted condition labels. $p$ values were obtained by taking the proportion of random tests with a test statistic equal or superior to the original value and were corrected for multiple comparisons using cluster-based correction. Channel clusters were determined by including each channel's immediate neighbors using triangulation (on average 6.7 neighbors) and cluster statistics were obtained by summing the $t$ scores of neighboring channels exceeding the critical value $(p<0.05)$ (for the detailed procedure, see Maris and Oostenveld, 2007).

ROI analyses were performed to compare the observed effects to previous studies investigating the alpha mu rhythm suppression during action observation and execution. In line with these previous studies, 10 central channels (C1-2-3-4-z, CP1-2-3-4-z) were selected for further analyses. An equivalent number of occipital channels (PO3-4-7-8-9$10-z, O 1-2-z)$ were selected to serve as the control occipital site where no crossmodal effects were expected. The main effect of each experimental condition was calculated by taking the difference between the two levels of each of the conditions (Transitive-Intransitive, Vibration on-Vibration off, Whole-hand grip-Precision grip). These differences were en- 
tered into a three-way repeated-measures ANOVA to investigate the effects of Modality (Execution, Observation), Location (Central, Occipital), and Condition (Transitivity, Vibration and Action).

Multivariate pattern classification. A linear support vector machine classifier was used to perform a fivefold cross-validated classification on all trials. Subsets of trials were created for classifier input by dividing the data into five independent chunks for each modality (for a total of 10 chunks), which were balanced to ensure an equal number of trials for each condition tested. A leave-one-chunk-out cross-validation was performed in which four chunks were used to train the classifier, which was then tested on an independent chunk. For each participant and classification, activity within each trial was normalized across all trials in the training set using a $z$-score transformation, and the same normalization parameters were used to normalize trials of the testing set. For each classification, all trials in one modality were included because all trials belonged to one of the two levels of each experimental condition. With this approach, at least 135 trials were used for classification (mean = $152.36, \mathrm{SD}=6.32$, range 135-160) depending on the number of trials left after artifact rejection. There was no significant difference in the number of trials included in each condition as confirmed with a 2 (Execution, Observation) $\times 3$ Condition (Vibration, Action type, Transitivity) repeated-measures ANOVA performed on the number of trials left after artifact rejection (all $p$ values $>0.60$ ).

Classification was first performed within modality (unimodal classification) to ensure that the mu rhythm response for each condition was distinguishable within modality. For the unimodal classification analysis, the classifier was trained and tested on trials of the same modality (Execution or Observation). Then, for the crossmodal classification analysis, the classifier was trained on four chunks from one modality and tested on a chunk of trials of the opposite modality. This procedure was repeated five times for each modality, condition, and location. The mean crossmodal classification accuracies for each modality as well as Friedman tests performed on the classification accuracies in the clusters of interest suggested a similar pattern of results for both modalities. The accuracies obtained were thus averaged across modalities to obtain one classification accuracy for each participant, condition, and location for both unimodal and crossmodal classifications.

At the channel level, the classifier was trained to use the three dimensions of the data; that is, time $(0-3 \mathrm{~s}$ in bins of $50 \mathrm{~ms} ; 61$ time bins), frequency $(8-13 \mathrm{~Hz}$ or $15-25 \mathrm{~Hz}$, in bins of $1 \mathrm{~Hz} ; 6$ or 11 frequency bins), and location (on average 6.7 channels in the neighborhood structure for searchlights or 10 channels in the clusters of interest for ROI analysis), to discriminate between the two levels of each main effect (Transitive vs Intransitive, Precision grip vs Whole-hand grip, Vibration on vs Vibration off). For the whole-scalp spatial searchlight analysis, the same neighborhood structure as used in the univariate analysis was used. Classification was thus performed at each channel using all time-frequency information from this channel and its neighbors (on average 6.7 neighbors) (for a similar approach but in time-frequency-sensor space using MEG, see Tucciarelli et al., 2015; Turella et al., 2016). This resulted in classification accuracy maps showing classification accuracy at each channel for each condition and participant. Maps in each condition were submitted to a one-sample $t$ test against chance accuracy (50\%) at the group level, and the significance of this test was assessed using the same permutation procedure used for univariate whole-scalp analysis (see Univariate analyses). For the ROI analyses at the channel level, the same time-frequency dimensions were used, but the classifier was applied separately on two clusters of 10 central channels and 10 occipital channels of interest. Classification accuracy in each condition and location was compared against chance using a Wilcoxon signed rank test contrasting classification performance with chance accuracy of 0.5 (Carlson et al., 2013; Ritchie et al., 2015). The main effect of Condition (Vibration, Action type, Transitivity) was assessed separately at the central and occipital channels using the Friedman test of differences.

At the source level, the classifier was trained to discriminate between the two levels of each main condition by using the spatial pattern of source activity. A spatial searchlight approach was used by building a neighborhood structure using all grid points within a sphere with a radius of $2 \mathrm{~cm}$ from each grid point (on average 28.6 neighbors). Classi- fication was then performed at each grid point and its neighbors. Classification accuracies in source space were projected to a standard MNI template for visualization. Source accuracy maps in each condition were submitted to a one-sample $t$ test against chance accuracy (50\%) at the group level, and the significance of this test was assessed using the same permutation procedure used for univariate whole-scalp analysis (see Univariate analyses).

\section{Results}

\section{Catch trials accuracy}

The average detection accuracy was $97.40 \%$ (SD $=3.18 \%$, range $=91 \%-100 \%)$ indicating that participants correctly identified the presence of the catch trial cue on the majority of presentations.

\section{Univariate analyses}

Scalp distribution of the mu rhythm suppression in the alpha band as well as a time-frequency representation of this suppression in each cluster of interest are shown in Figure 3. The wholescalp analyses of the alpha mu rhythm suppression performed in the observation modality revealed significant main effects of Transitivity at a central left cluster of channels indicating stronger mu suppression for the observation of transitive movements relative to the observation of intransitive movements. No significant main effects of Vibration and Action type were found during observation. The same analyses performed in the execution modality showed significant main effects of Vibration and Transitivity. These effects indicated significantly stronger suppression for Vibration on trials relative to Vibration off trials in a large frontal-right cluster of channels as well as significantly stronger suppression for executed intransitive trials relative to transitive trials in a cluster of left central and parieto-occipital channels. No significant main effect of Action type was found during execution.

Mu rhythm suppression at each level of the three main experimental conditions at the central and occipital clusters of interest are shown in Figure 5A. The three-way repeated-measures ANOVA revealed a significant Modality $\times$ Location interaction $\left(F_{(1,19)}=\right.$ $\left.5.03, p=0.037, \eta_{\mathrm{p}}^{2}=0.21\right)$, indicating that the overall effect of the experimental conditions was stronger at the central relative to the occipital location in the observation modality but not in the execution modality. There was also a significant Modality $\times$ Type interaction $\left(F_{(2,38)}=5.70, p=0.012, \eta_{\mathrm{p}}^{2}=0.23\right)$ due to the fact that, in the Transitive condition, transitive trials led to a stronger mu suppression relative to intransitive trials during observation, but the opposite effect was present during execution. There was no significant main effects of Modality, Location, or Type and no other interaction reached significance (all $p$ values $>0.05$ ).

Scalp distribution of the mu-rhythm suppression in the beta band as well as a time-frequency representation of this suppression in each cluster of interest are shown in Figure 4. The wholescalp analyses of the beta rhythm suppression performed in the observation modality revealed significant main effects of Vibration in a large cluster of channels over the posterior left hemisphere, indicating stronger beta suppression for the observation of movements with a concurrent vibration compared with the observation of movements without concurrent vibration. The same analyses performed in the execution modality did not show any univariate difference between the conditions.

Mu-rhythm suppression in the beta band at each level of the three main experimental conditions at the central and occipital clusters of interest are shown in Figure $5 B$. The three-way repeated-measures ANOVA revealed a significant Modality $\times$ 


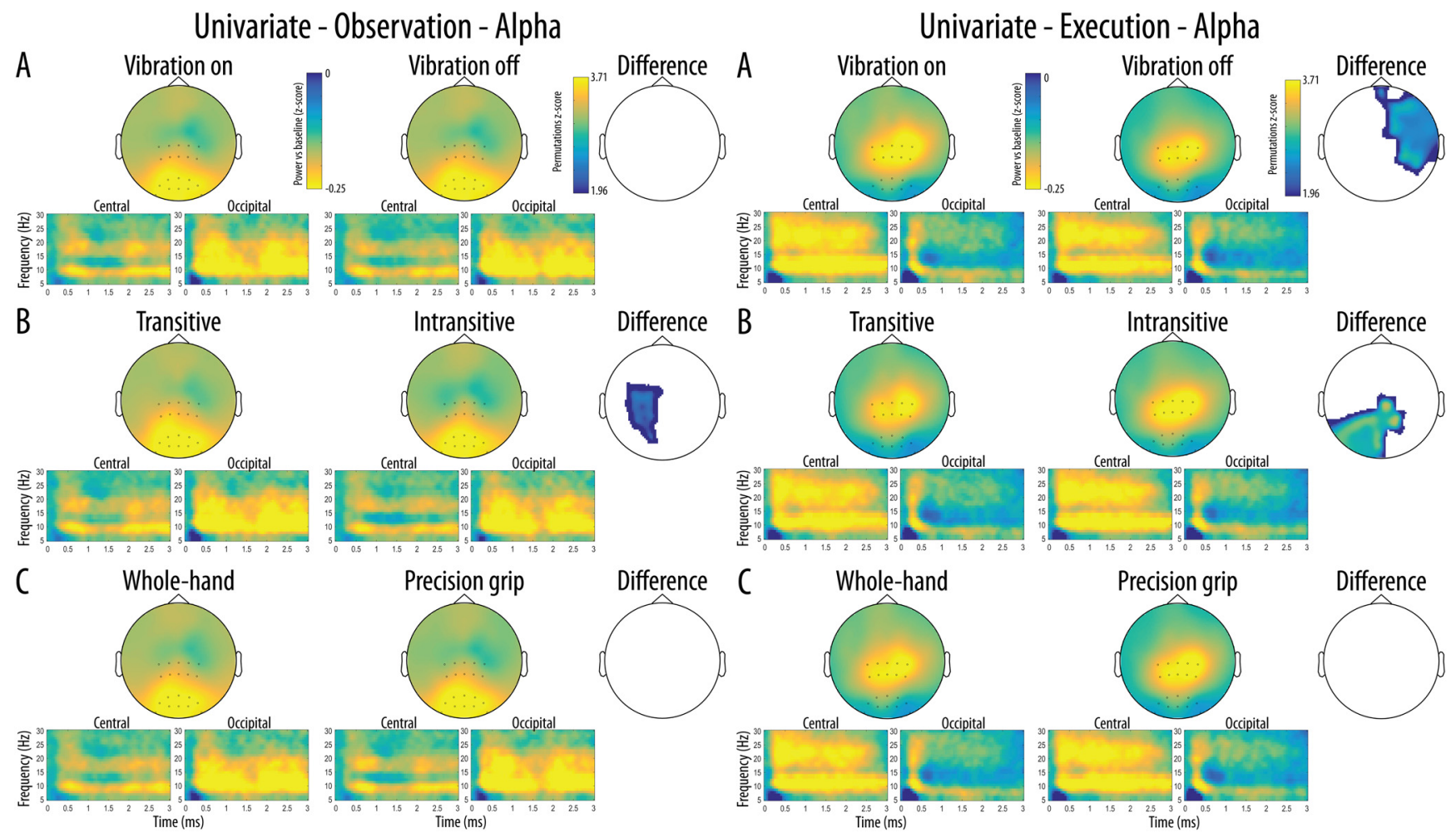

Figure 3. Scalp distribution of the alpha mu rhythm suppression relative to baseline for the two levels of each Condition (A, Vibration; $\boldsymbol{B}$, Transitivity; $\boldsymbol{C}$, Action type) as a function of Modality (Left, Execution; Right, Observation). The difference maps show clusters of channels with significant main effects surviving correction for multiple comparisons for each Condition and Modality. Time-frequency plots show the time course of frequency activity at the central and occipital clusters of interest. Channels included in these clusters are marked on the scalp maps.

Univariate - Observation- Beta
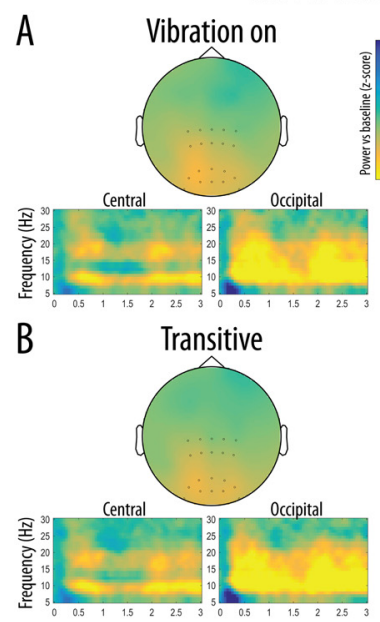

C
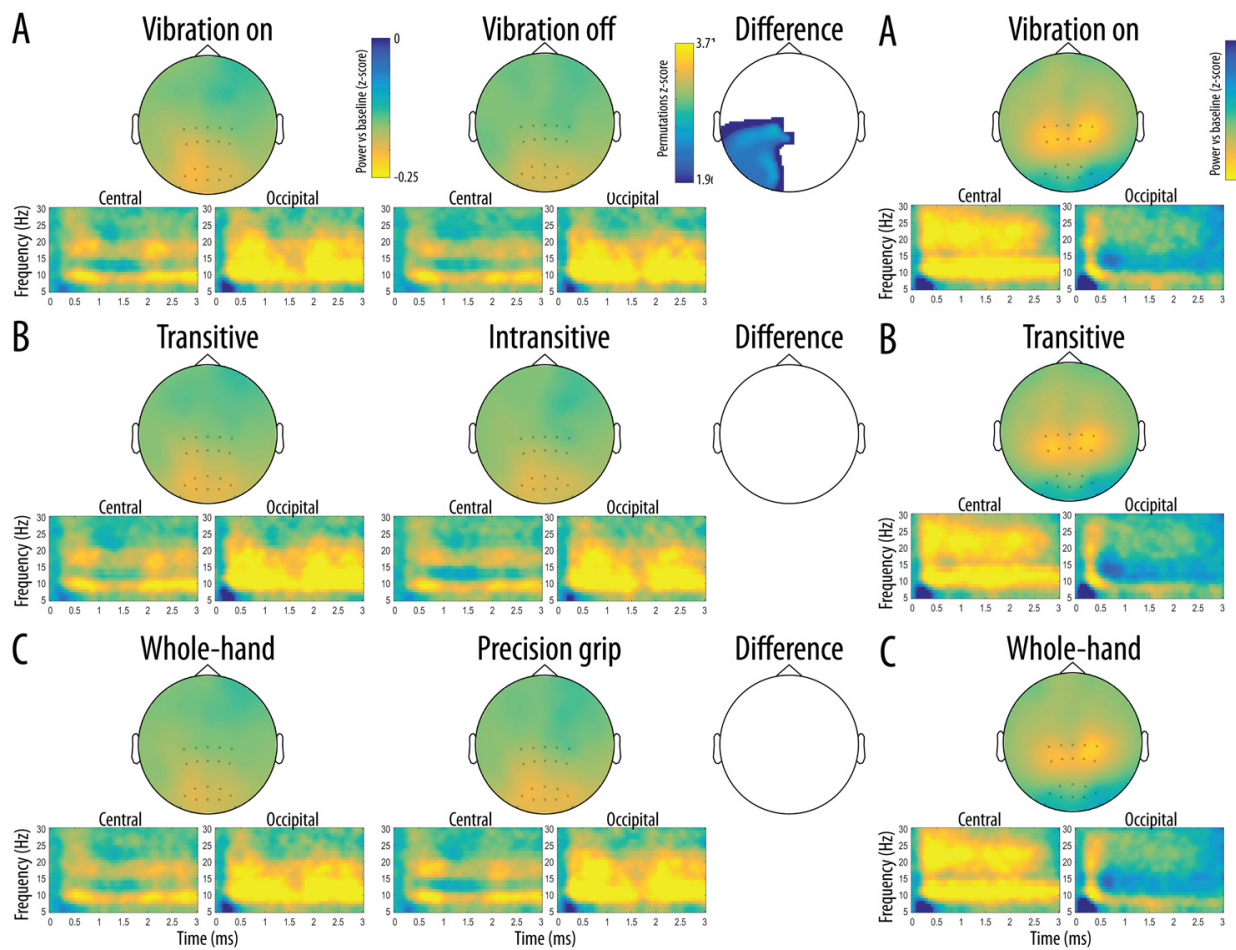

Precision grip
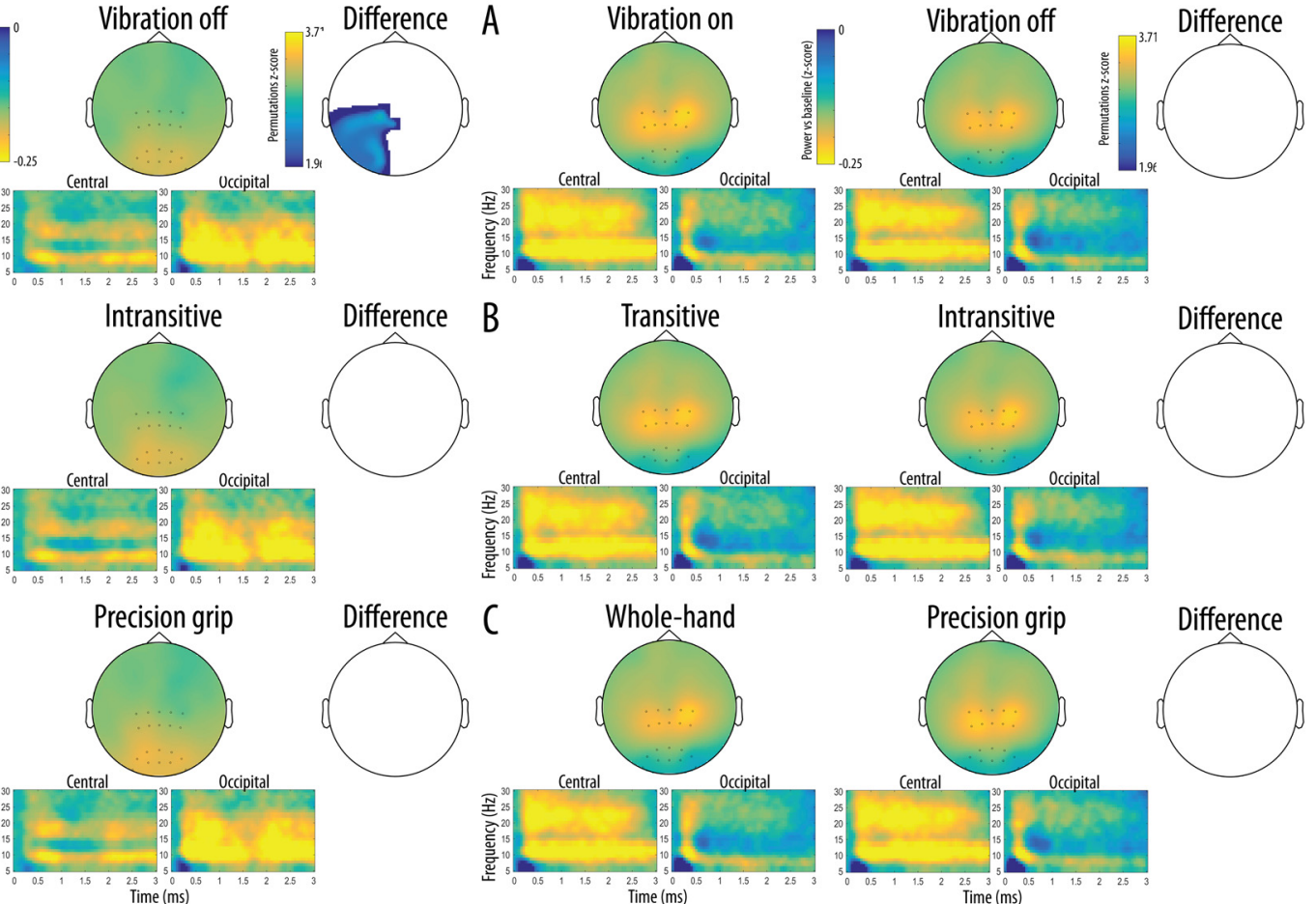

Figure 4. Scalp distribution of the beta suppression relative to baseline for the two levels of each Condition ( $\boldsymbol{A}$, Vibration; $\boldsymbol{B}$, Transitivity; $\boldsymbol{C}$, Action type) as a function of Modality (Left, Execution; Right, Observation). The difference maps show clusters of channels with significant main effects surviving correction for multiple comparisons for each Condition and Modality. Time-frequency plots show the time course of frequency activity at the central and occipital clusters of interest. Channels included in these clusters are marked on the scalp maps. 
A
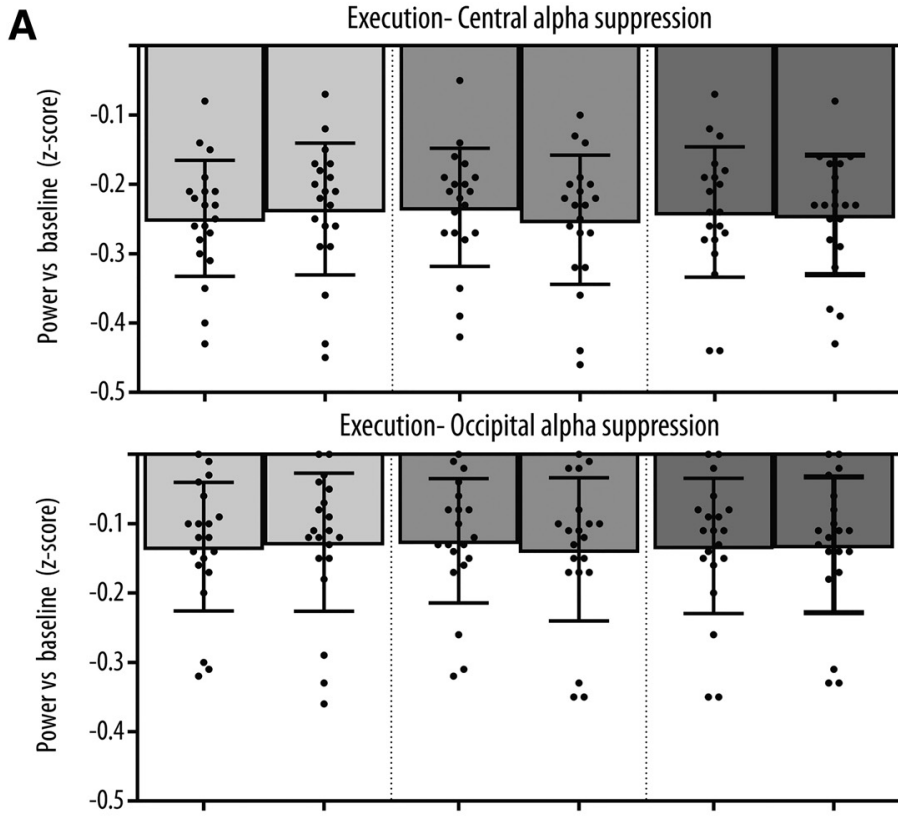

B
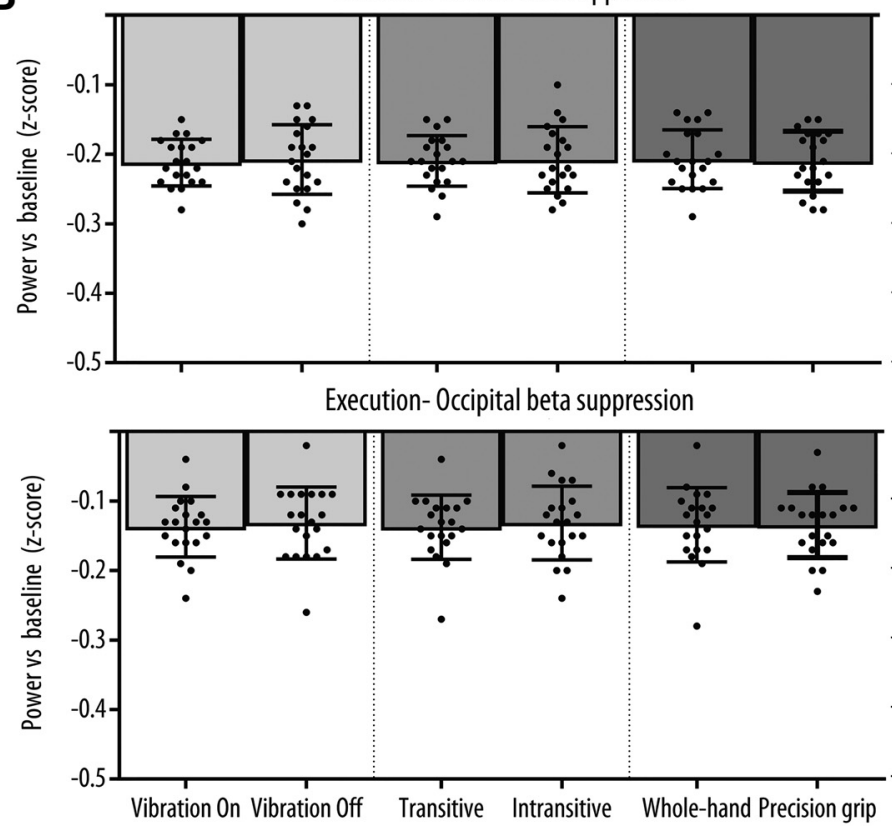

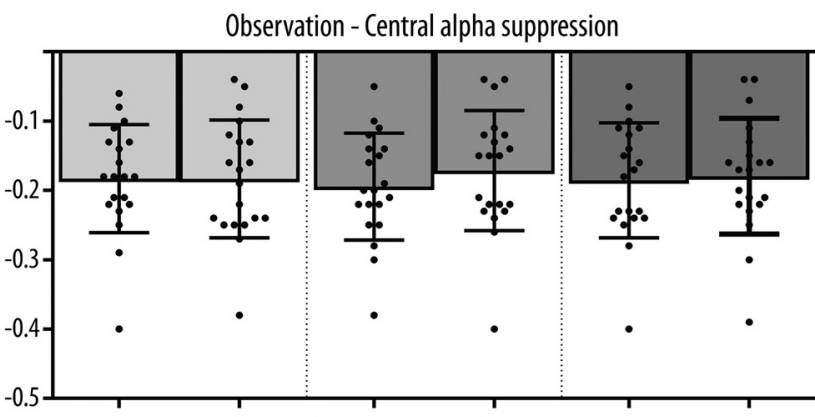

Observation - Occipital alpha suppression

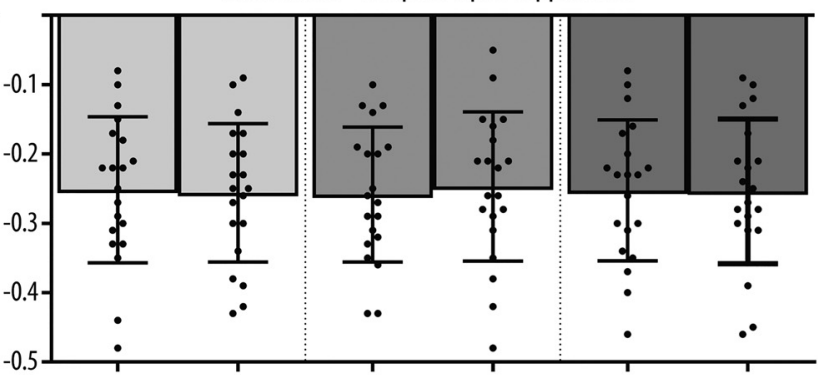

Observation - Central beta suppression

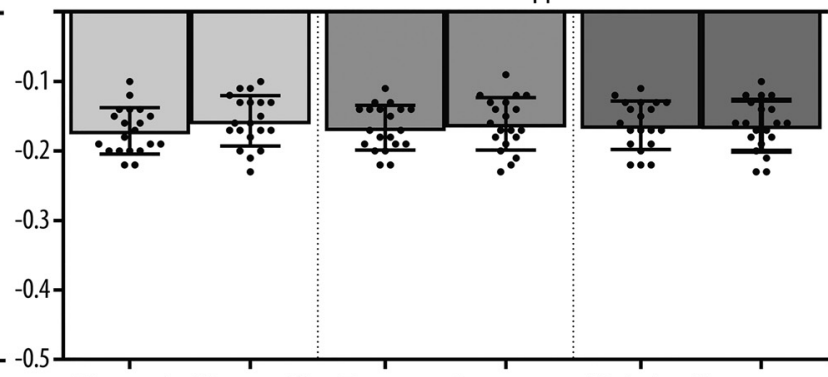

Observation - Occipital beta suppression

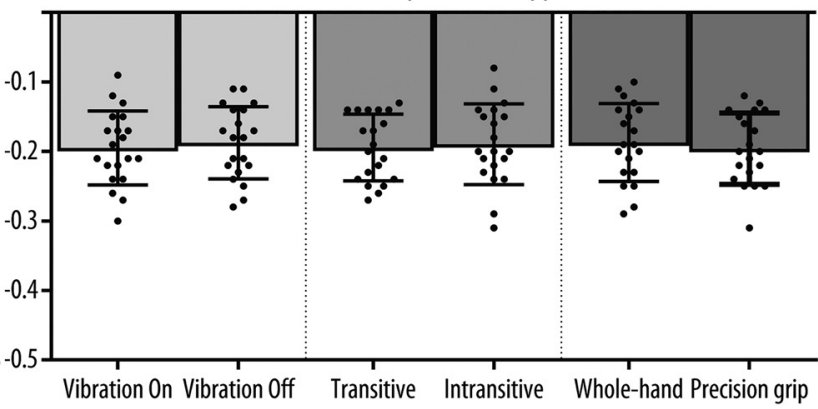

Figure 5. Mean $(\boldsymbol{A})$ alpha and $(\boldsymbol{B})$ beta suppression relative to baseline for the two levels of each Condition (Vibration, Transitivity, Action type) as a function of Modality (Left, Execution; Right, Observation) and Location (Left column, Central; Right column, Occipital). Error bars indicate $95 \%$ Cl. Black dots indicate the mean suppression for each subject.

Location interaction $\left(F_{(1,19)}=5.78, p=0.027, \eta_{\mathrm{p}}^{2}=0.23\right)$ indicating that the overall effect of the experimental conditions on beta suppression was stronger for the execution modality relative to observation at the central location, whereas the opposite effect of modality was observed at the occipital location. There was no significant main effects of Modality, Location, or Type, and no other interaction reached significance (all $p$ values $>0.05$ ).

\section{Multivariate pattern classification}

\section{Unimodal classification}

As shown in Figure $5 A$, the spatial searchlight analysis performed at the channel level revealed widespread above-chance unimodal classification accuracy across all channels for the three experimental conditions in both the alpha and beta bands. As shown in Figure $5 B$, classification at the source level for the alpha band suggested that widespread sources mainly located in the frontal and parietal areas were responsible for the unimodal classification in all three conditions. Permutation analyses indicated that all these sources showed significantly above-chance classification. This was reflected in the ROI analyses in which Wilcoxon signed-rank tests revealed significantly above-chance classification accuracy for all conditions at both the central and occipital electrode clusters (for $p$ values, see Fig. 6C). Friedman tests indicated that there was a significant effect of Condition at the central cluster $\left(\chi_{(2)}^{2}=6.40, p=0.041\right)$ due to a significantly higher unimodal classification accuracy in the Transitivity compared with the Action type manipulation $(p=0.037)$. There was no significant effect of Condition at the occipital cluster $\left(\chi_{(2)}^{2}=2.45\right.$, $p=0.293$ ). For the beta band, sources mainly located in the 


\section{Multivariate - Unimodal classification - Alpha}

A
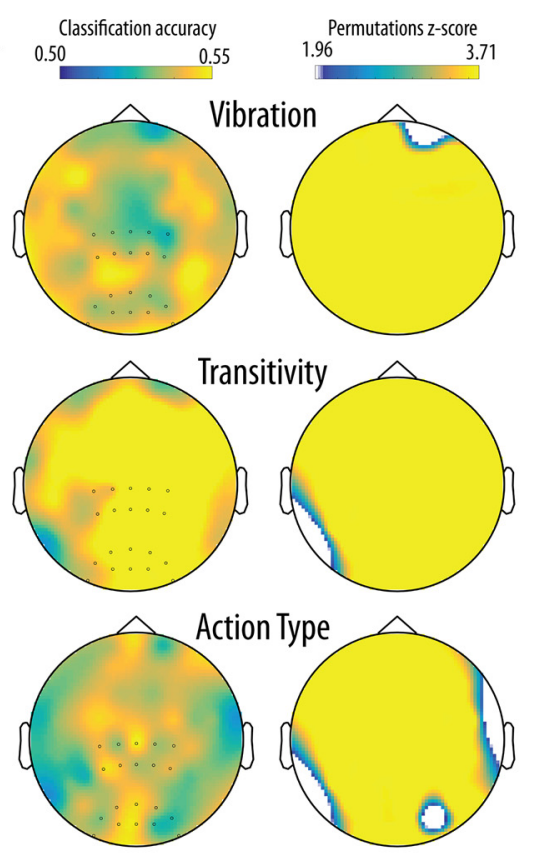

B
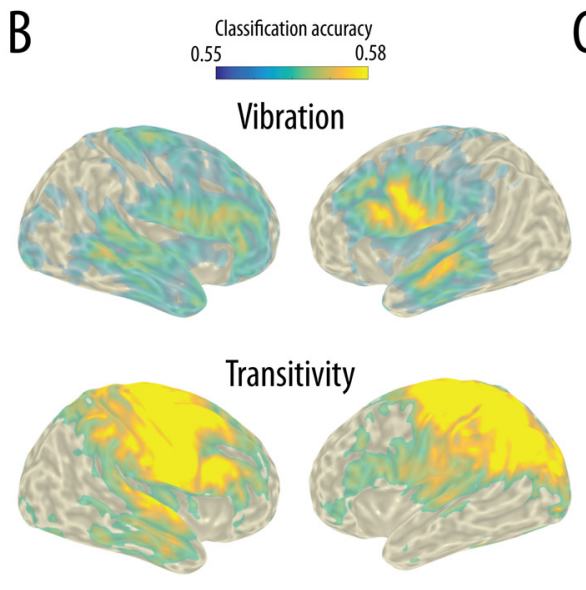

Transitivity

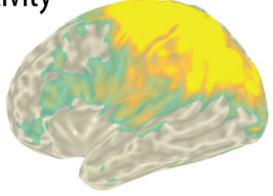

Action Type

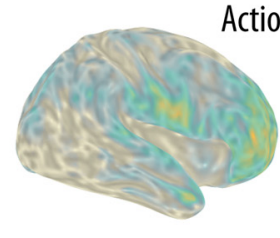

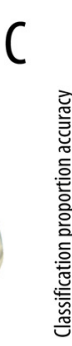
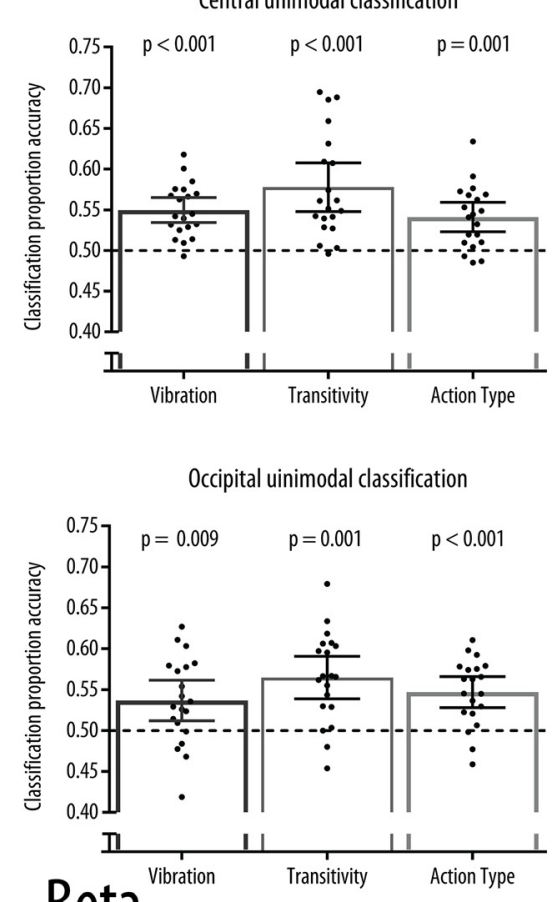

\section{Multivariate - Unimodal classification - Beta}
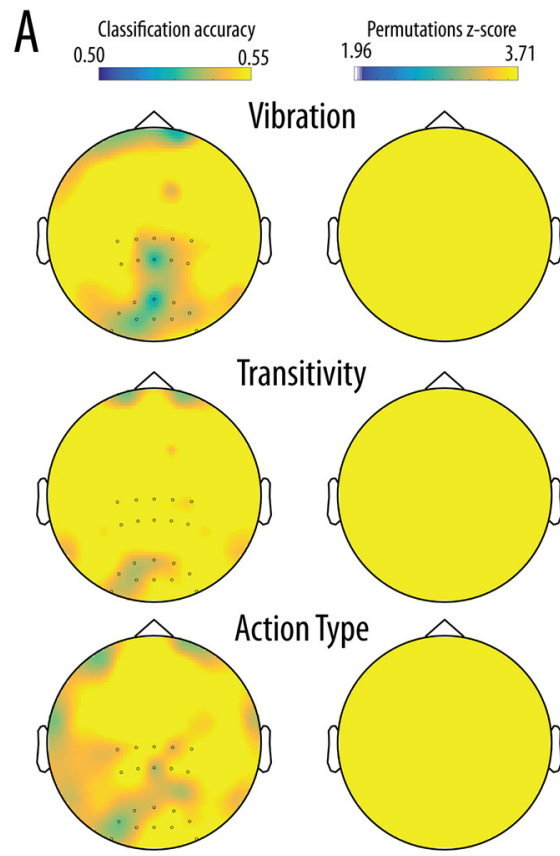

B

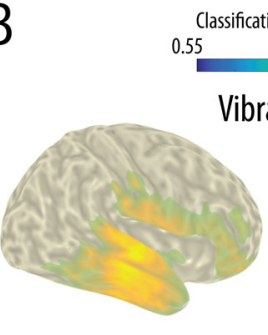

Transitivity
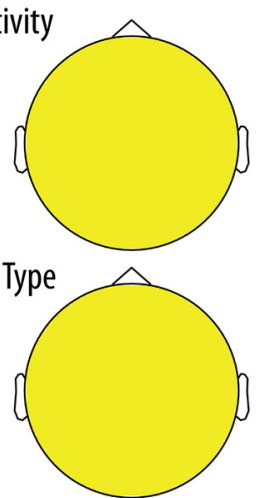
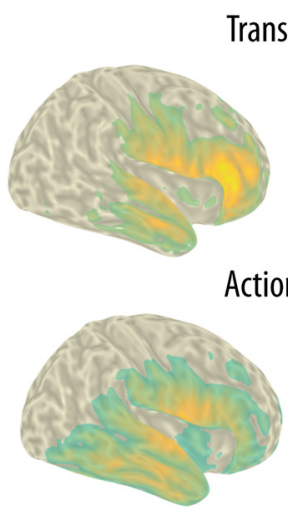

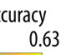

Vibration
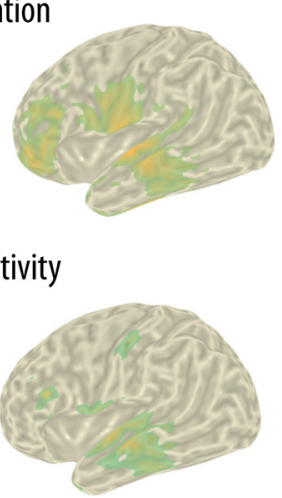

Action Type

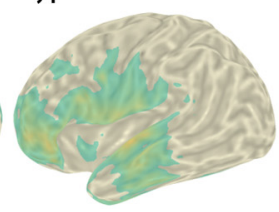

C
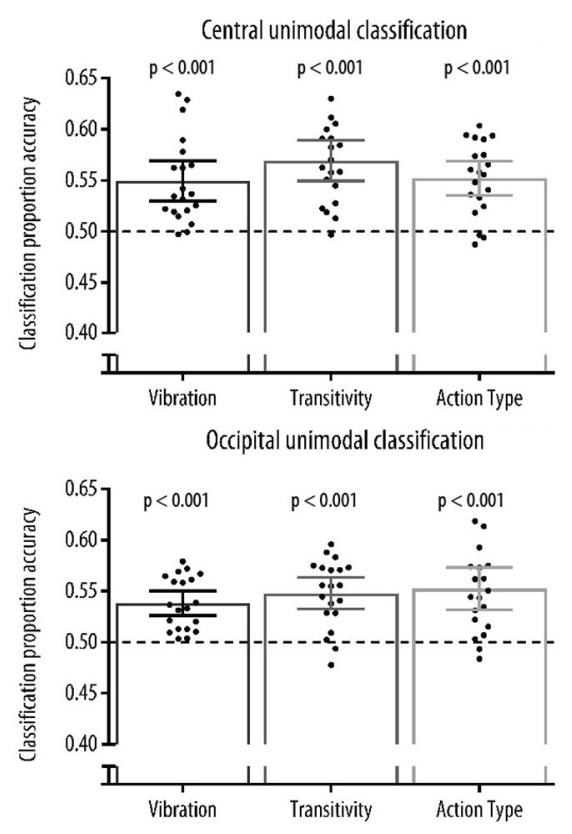

Figure 6. Results for the multivariate unimodal classification for the alpha (top) and beta (bottom) bands. A, Results from the searchlight analyses at the channel level and maps showing cluster of channels with classification accuracy significantly above chance $(0.50)$ and surviving correction for multiple comparisons. B, Classification accuracy for the searchlight analyses performed at the source level. Only grid points with accuracy above the $95 \%$ of the maximum accuracy were projected to the scalp for visualization purposes. All grid points projected show significantly above chance accuracy. C, Mean and distribution of classification accuracy as a function of Condition and Location for the classification performed in the central and occipital clusters of interest. Dotted line indicates chance classification accuracy (0.5). Error bars indicate $95 \%$ Cl. Black dots indicate the mean classification accuracy for each participant.

frontal and temporal areas showed significantly above chance classification and were responsible for the unimodal classification in all three conditions. ROI analyses using Wilcoxon signed-rank tests revealed significantly above-chance classification accuracy for all conditions at both the central and occipital electrode clusters (for $p$ values, see Fig. $6 C$ ). Friedman tests indicated that there was no significant effect of Condition at the central $\left(\chi_{(2)}^{2}=1.80, p=0.091\right)$ or occipital cluster $\left(\chi_{(2)}^{2}=1.30, p=0.522\right)$.

Crossmodal classification

For the alpha band, the spatial searchlight analysis performed at the channel level revealed clusters of channels showing above- 


\section{Multivariate - Crossmodal classification - Alpha}
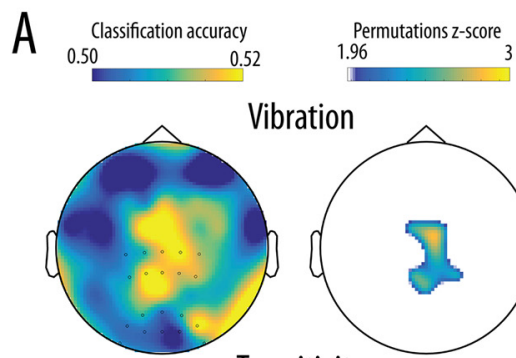

Vibration

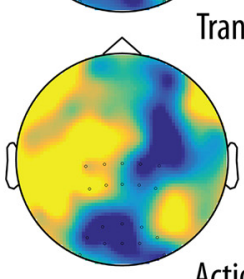

Transitivity
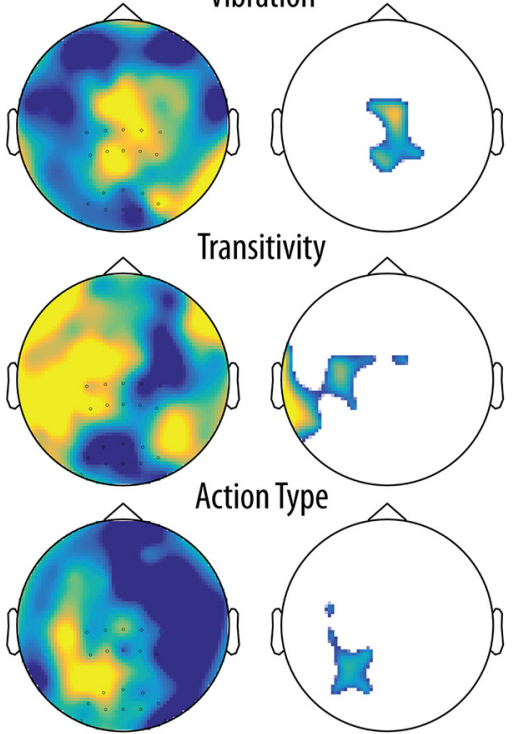

B
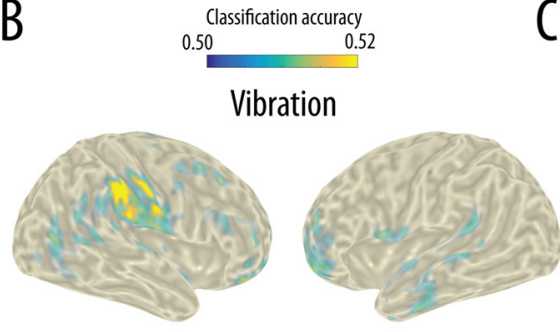

Transitivity

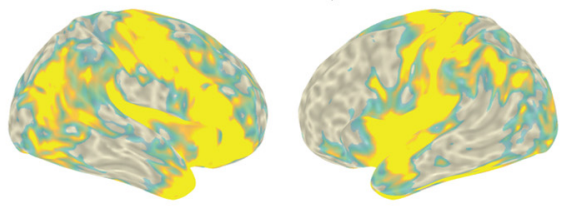

Action Type
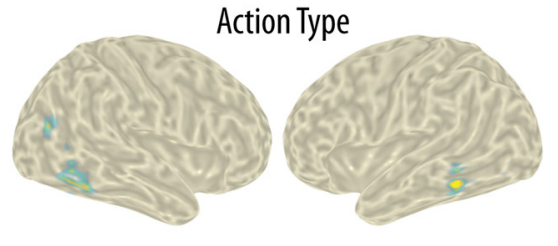

Central crossmodal classification

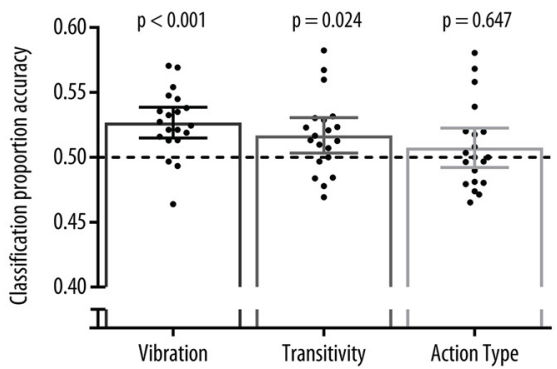

Occipital crossmodal classification

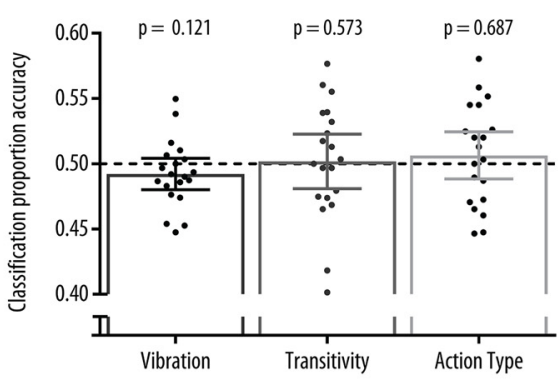

\section{Multivariate - Crossmodal classification - Beta}
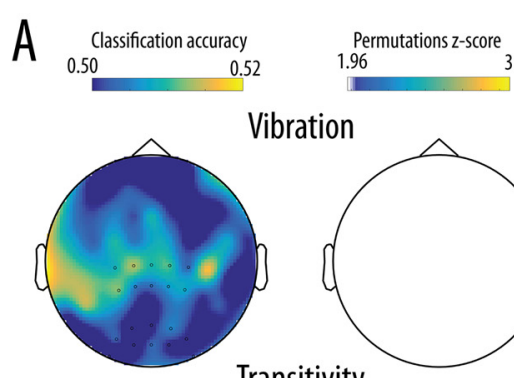

Vithation

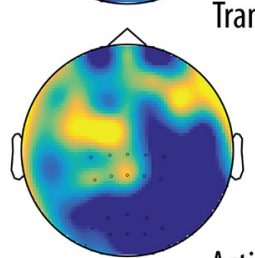

Transitivity
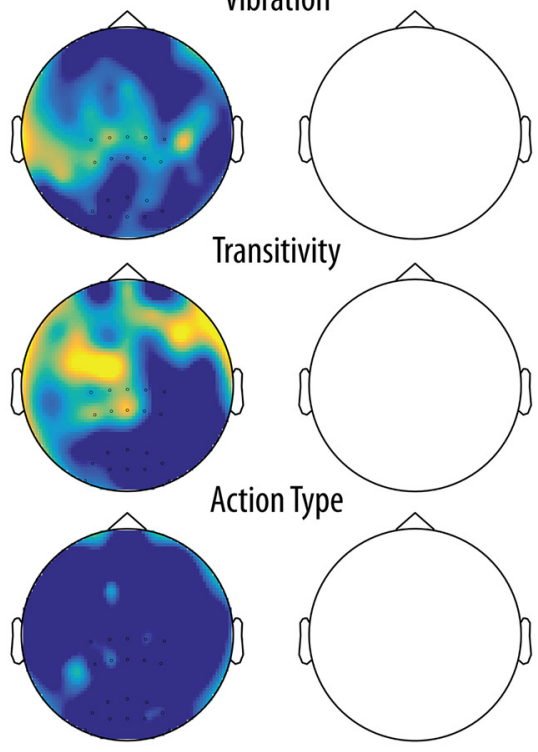

B
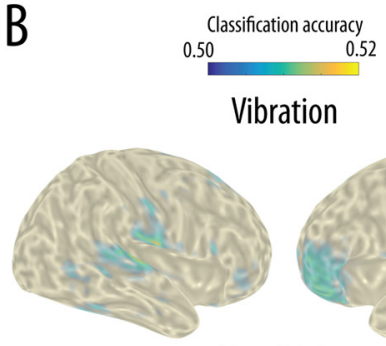

Transitivity

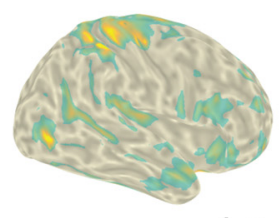

Action Type
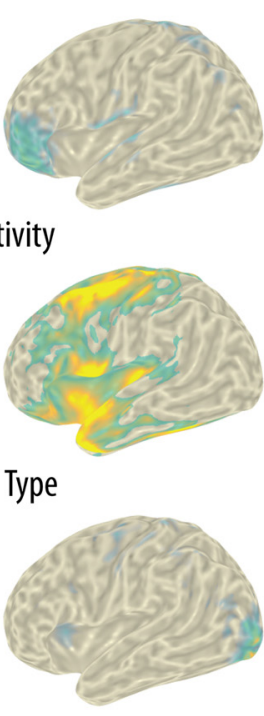

Central crossmodal classification

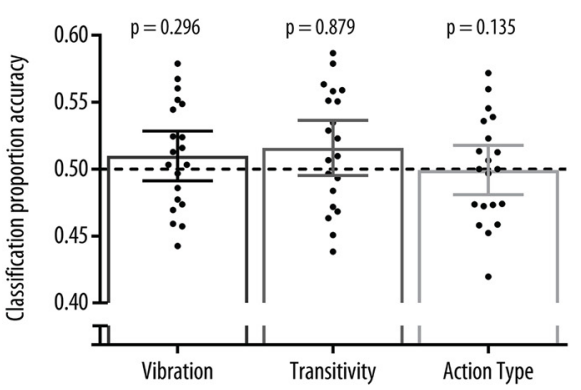

Occipital crossmodal classification

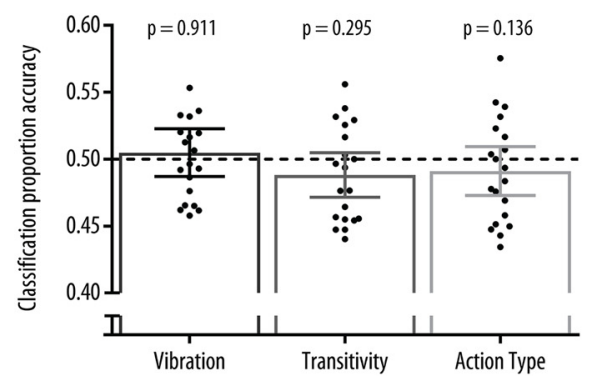

Figure 7. Results for the multivariate unimodal classification for the alpha (top) and beta (bottom) bands. A, Results from the searchlight analyses at the channel level and maps showing clusters of channels with classification accuracy significantly above chance $(0.50)$ and surviving correction for multiple comparisons. $B$, Classification accuracy for the searchlight analyses performed at the source level. Only grid points with accuracy above the $95 \%$ of the maximum accuracy were projected to the scalp for visualization purposes. Crossmodal classification at the source level is illustrated for visualization purposes only as no grid points showed significantly above chance accuracy. C, Mean and distribution of classification accuracy as a function of Condition and Location for the classification performed in the central and occipital clusters of interest. Dotted line indicates chance classification accuracy (0.5). Error bars indicate $95 \%$ Cl. Black dots indicate the mean classification accuracy for each participant.

chance crossmodal classification accuracy for the three experimental conditions (Fig. 7A). For the Vibration condition, this cluster covered mainly central channels. For the Transitivity condition, the significant cluster covered left central and temporal channels. A cluster of left parieto-occipital channels showed above-chance classification in the Action type condition. The crossmodal classification accuracy at the source level is shown in Figure $7 B$ for visualization purposes; the permutation analyses indicated that classification was not significantly above chance at the source level. Regardless of significance, source level analyses 
suggested that, for the Vibration condition, a right parietal cluster partly covering the somatosensory cortex contributed most to the crossmodal classification. In the Transitivity condition, sources generating the crossmodal classification were widely distributed mainly over frontoparietal areas. Finally, for the Action type condition, small clusters located over temporal and occipital areas showed above-chance crossmodal classification. As shown in Figure $7 C$, the ROI analyses revealed that significantly above-chance crossmodal classification accuracy was reached only in the Vibration and Transitivity conditions and only at the central cluster. This was confirmed by Friedman tests showing a significant main effect of Condition at the central cluster $\left(\chi_{(2)}^{2}=9.10, p=0.011\right)$ but not at the occipital cluster $\left(\chi_{(2)}^{2}=0.90, p=0.638\right)$. At the central cluster, this effect was due to significantly higher classification in the Vibration condition compared with the Action type condition ( $p=0.025)$, while there was no other pairwise difference between the conditions (all $p$ values $>0.18$ ).

The crossmodal classification performed in the beta band did not indicate any significantly above-chance classification in the searchlight analysis performed at the channel level (Fig. 7A), at the source level (Fig. 7B), or in the ROI analyses (Fig. 7C).

\section{Discussion}

The present study examined the validity of the claim that the EEG mu rhythm is a valid index of MN activity by testing for the presence of crossmodal specificity in response to observed and executed actions. Moreover, it assessed the alternative prediction that the mu rhythm demonstrates crossmodal and specific responses to the observation and receipt of tactile stimulation. This was achieved using a multivariate crossmodal classification approach to test whether the central mu rhythm contains sufficient crossmodal information to discriminate between two different types of actions, between the presence or absence of tactile stimulation, and between transitive and intransitive actions.

Results from the crossmodal classification of mu rhythm response at the channel level were as predicted by the tactile stimulation account, and support the idea that the central mu rhythm shows crossmodal specificity primarily for the somatosensory features of observed and executed action. Although exploratory searchlight analyses indicated significant crossmodal classification for all conditions, central channels contributed mostly to the classification of conditions showing strong variation in tactile features. Crossmodal classification of action type was achieved for alpha-band activity that is not central, and not likely to be reflective of mirror neuron system activity. Mu suppression experiments investigating mirror neuron processes commonly consider changes in activity at the central sites to be reflective of mirror neuron system activity. The results from the ROI analyses in the current study strongly suggest that responsivity at these sites is not in keeping with mirror neuron accounts of central mu suppression. This was supported by a priori region of interest analyses performed at a cluster of central channels, which revealed above-chance crossmodal classification only for the tactile stimulation and transitivity conditions, and significantly higher classification accuracy for the presence of tactile stimulation relative to the type of action. The same analysis performed at the control occipital channels did not indicate any significant classification. The crossmodal specificity of the mu rhythm to somatosensory features of actions suggests that the central mu rhythm response to action observation and execution observed in the current and previous studies might be better explained by sensory processing rather than motor mirroring (Dinstein et al., 2008; Cook et al., 2014; Coll et al., 2015). It should be noted however, that the preceding studies reported the results of univariate analyses, rather than multivariate analyses.

Although this is, as far as we are aware, the first study to investigate the crossmodal specificity of mu rhythm responses using multivariate classification, it is not the first to suggest that the crossmodal mu rhythm response indexes somatosensory features of action rather than action type (Coll et al., 2015). The association of the mu rhythm with sensory processing has also been demonstrated by several previous studies. The central alpha mu rhythm is known to be modulated by somatosensory attention (Jones et al., 2010; Anderson and Ding, 2011), and prestimulation mu rhythm activity can reliably predict the detection of a somatosensory stimulus (Linkenkaer-Hansen and Nikulin, 2004). Previous studies using fMRI or source localization also indicate that the mu rhythm can be associated with the activity of the somatosensory cortices (Hari et al., 1998; Cheyne et al., 2003; Ritter et al., 2009; Arnstein et al., 2011) and is responsive to the observation of tactile stimulation (Muthukumaraswamy and Johnson, 2004; Coll et al., 2015). In line with this previous research, we found above-chance crossmodal classification accuracy when the classifier was used to discriminate between the presence or absence of tactile stimulation in the self or in the other in central channels, and this accuracy was significantly higher than for classification of action types. The source analyses performed in the current experiment did not reveal any significantly above-chance crossmodal classification at the source level. This should be interpreted with caution given that the relatively sparse EEG montage used and the lack of individual anatomical information make these statistical analyses highly conservative. The visualization of crossmodal classification accuracy at the source level nevertheless suggests that crossmodal classification of the mu rhythm response to tactile stimulation and transitivity was driven by frontoparietal sources, including somatosensory areas.

The unimodal classification results obtained in the current study suggest that the unimodal mu rhythm response shows little specificity. Indeed, classifiers trained and tested on trials of the same modality showed widespread above-chance classification at both channel and source levels. Indirect evidence for the lack of spatial and functional specificity of the mu rhythm response is also present in a recent meta-analysis of mu rhythm suppression studies. Fox et al. (2016) analyzed 85 studies and found that, across these studies, mu rhythm suppression did not show many of the properties of $\mathrm{MN}$ activity, such as preference for objectdirected movement or biological motion. In addition, the effect size of alpha suppression compared with baseline during action observation was not found to be greater at central electrodes compared with occipital electrodes, suggesting that the contribution of the occipital alpha rhythm might explain many of the effects reported in the literature (Fox et al., 2016; Hobson and Bishop, 2016).

To compare the results obtained from the multivariate pattern classification to the results obtained in previous studies, we also performed a univariate analysis of mu rhythm suppression relative to baseline for the same experimental conditions by averaging activity over all time-frequency bins. When comparing the average mu rhythm suppression relative to baseline, we found similar suppression effects in terms of effect size and location compared with previous studies (for a meta-analysis, see Fox et al., 2016). However, this analysis showed that alpha rhythm suppression was not specific to the central electrodes and was relatively insensitive to differences between conditions. These results suggest that the analytical approach used in previous research is 
inadequate to detect the specificity of crossmodal mu rhythm responses and is insensitive to subtle differences between conditions. This was to be expected considering that, by averaging over all features of the data, this approach does not take into account differences in multivariate patterns that can differ between conditions and participants. This is also in line with a recent high-powered preregistered report indicating that mu rhythm suppression effects found using this analytical approach are weak and unreliable (Hobson and Bishop, 2016).

Although not the primary focus of the study, activity in the beta band was submitted to the same analyses as the alpha mu rhythm. Beta activity has also been previously associated with MN activity, although less frequently than the alpha mu rhythm (Rossi et al., 2002; Muthukumaraswamy and Singh, 2008). Here, beta activity was shown to contain unimodal information on the different experimental conditions. This is in line with previous studies using a similar analytical approach showing that beta activity can be used to classify observed (Tucciarelli et al., 2015) or executed actions (Turella et al., 2016). However, the crossmodal classification of beta activity was at chance level in all conditions. The current results therefore suggest that beta rhythm suppression during action observation and action execution does not show crossmodal action specificity.

Limitations to this study need to be acknowledged. First, it should be noted that the crossmodal classification approach used in the current study could be quite conservative, and that it might therefore lack the sensitivity to detect central crossmodal mu rhythm responses to the motor features of the observed actions. It should also be noted that EEG activity represents a superposition of the activity of large neuronal populations and channel level analyses might lack the spatial specificity to demonstrate crossmodal classification of weaker effects. Therefore, even though crossmodal classification at the central channels was clearly higher for sensory features of actions, the absence of crossmodal classification for action types cannot be interpreted as the absence of crossmodal specificity for observed and executed actions in the mu rhythm response.

In conclusion, we have shown that the central alpha mu rhythm shows crossmodal specificity primarily for the observation and receipt of a tactile stimulation and that multivariate pattern classification is more sensitive to subtle differences between conditions than univariate analyses. This is, to our knowledge, the first study to use multivariate pattern classification to assess the crossmodal specificity of EEG responses. Combined with other sources of evidence, they question the appropriateness of mu rhythm suppression as a measure of MN activity and suggest that multivariate crossmodal analyses are needed to adequately study this relationship in the future. This study and others support the idea that a new framework is needed to explain the significance of the central mu rhythm for social perception in health and in disease, and that the search for this new framework should be directed away from a simplistic matching between $\mathrm{mu}$ rhythm suppression and $\mathrm{MN}$ activity and use methodologies that are able to take into account the multivariate nature of EEG data.

\section{References}

Anderson KL, Ding M (2011) Attentional modulation of the somatosensory mu rhythm. Neuroscience 180:165-180. CrossRef Medline

Arnstein D, Cui F, Keysers C, Maurits NM, Gazzola V (2011) Suppression during action observation and execution correlates with BOLD in dorsal premotor, inferior parietal, and SI cortices. J Neurosci 31:14243-14249. CrossRef Medline

Bernier R, Dawson G, Webb S, Murias M (2007) EEG mu rhythm and imi- tation impairments in individuals with autism spectrum disorder. Brain Cogn 64:228-237. CrossRef Medline

Bernier R, Aaronson B, McPartland J (2013) The role of imitation in the observed heterogeneity in EEG mu rhythm in autism and typical development. Brain Cogn 82:69-75. CrossRef Medline

Carlson T, Tovar DA, Alink A, Kriegeskorte N (2013) Representational dynamics of object vision: the first $1000 \mathrm{~ms}$. J Vis 13:1-19. CrossRef Medline

Cheng Y, Lee PL, Yang CY, Lin CP, Hung D, Decety J (2008) Gender differences in the mu rhythm of the human mirror-neuron system. PLoS One 3:1-7. CrossRef Medline

Cheyne D, Gaetz W, Garnero L, Lachaux JP, Ducorps A, Schwartz D, Varela FJ (2003) Neuromagnetic imaging of cortical oscillations accompanying tactile stimulation. Cogn Brain Res 17:599-611. CrossRef Medline

Coll MP, Bird G, Catmur C, Press C (2015) Cross-modal repetition effects in the mu rhythm indicate tactile mirroring during action observation. Cortex 63:121-131. CrossRef Medline

Cook R, Bird G, Catmur C, Press C, Heyes C (2014) Mirror neurons: from origin to function. Behav Brain Sci 37:177-192. CrossRef Medline

Dinstein I, Thomas C, Behrmann M, Heeger DJ (2008) A mirror up to nature. Curr Biol 18:233. CrossRef Medline

di Pellegrino G, Fadiga L, Fogassi L, Gallese V, Rizzolatti G (1992) Understanding motor events: a neurophysiological study. Exp Brain Res 91: 176-180. CrossRef

Fox NA, Bakermans-Kranenburg MJ, Yoo KH, Bowman LC, Cannon EN, Vanderwert RE, Ferrari PF, van IJzendoorn MH (2015) Assessing human mirror activity with EEG mu rhythm: a meta-analysis. Psychol Bull 142:291-313. CrossRef Medline

Gallese V (2001) The roots of empathy: the 'shared manifold' hypothesis: from mirror neurons to empathy. J Conscious Stud 8:33-50. CrossRef Medline

Gallese V, Fadiga L, Fogassi L, Rizzolatti G (1996) Action recognition in the premotor cortex. Brain 119:593-609. CrossRef Medline

Gross J, Kujala J, Hamalainen M, Timmermann L, Schnitzler A, Salmelin R (2001) Dynamic imaging of coherent sources: studying neural interactions in the human brain. Proc Natl Acad Sci U S A 98:694-699. CrossRef Medline

Hari R, Forss N, Avikainen S, Kirveskari E, Salenius S, Rizzolatti G (1998) Activation of human primary motor cortex during action observation: a neuromagnetic study. Proc Natl Acad Sci U S A 95:15061-15065. CrossRef Medline

Hobson HM, Bishop DV (2016) Mu suppression: a good measure of the human mirror neuron system? Cortex 82:290-310. CrossRef Medline

Horan WP, Pineda JA, Wynn JK, Iacoboni M, Green MF (2014) Some markers of mirroring appear intact in schizophrenia: evidence from $\mathrm{mu}$ suppression. Cogn Affect Behav Neurosci 14:1049-1060. CrossRef Medline

Jones SR, Kerr CE, Wan Q, Pritchett DL, Hämäläinen M, Moore CI (2010) Cued spatial attention drives functionally relevant modulation of the mu rhythm in primary somatosensory cortex. J Neurosci 30:13760-13765. CrossRef Medline

Kilner JM, Lemon RN (2013) What we know currently about mirror neurons. Curr Biol 23:R1057-R1062. CrossRef Medline

Kriegeskorte N, Goebel R, Bandettini P (2006) Information-based functional brain mapping. Proc Natl Acad Sci U S A 103:3863-3868. CrossRef Medline

Linkenkaer-Hansen K, Nikulin VV, Palva S, Ilmoniemi RJ, Palva JM (2004) Prestimulus oscillations enhance psychophysical performance in humans. J Neurosci 24:10186-10190. CrossRef Medline

Maris E, Oostenveld R (2007) Nonparametric statistical testing of EEG- and MEG-data. J Neurosci Methods 164:177-190. CrossRef Medline

McCormick LM, Brumm MC, Beadle JN, Paradiso S, Yamada T, Andreasen N (2012) Mirror neuron function, psychosis, and empathy in schizophrenia. Psychiatry Res 201:233-239. CrossRef Medline

Moreno I, de Vega M, León I (2013) Understanding action language modulates oscillatory mu and beta rhythms in the same way as observing actions. Brain Cogn 82:236-242. CrossRef Medline

Muthukumaraswamy SD, Johnson BW (2004) Changes in rolandic mu rhythm during observation of a precision grip. Psychophysiology 41:152156. CrossRef Medline

Muthukumaraswamy SD, Singh KD (2008) Modulation of the human mirror neuron system during cognitive activity. Psychophysiology 45:896905. CrossRef Medline 
Oberman LM, Hubbard EM, McCleery JP, Altschuler EL, Ramachandran VS, Pineda JA (2005) EEG evidence for mirror neuron dysfunction in autism spectrum disorders. Cogn Brain Res 24:190-198. CrossRef Medline

Oberman LM, Ramachandran VS, Pineda JA (2008) Modulation of mu suppression in children with autism spectrum disorders in response to familiar or unfamiliar stimuli: the mirror neuron hypothesis. Neuropsychologia 46:1558-1565. CrossRef Medline

Oostenveld R, Fries P, Maris E, Schoffelen JM (2011) FieldTrip: Open source software for advanced analysis of MEG, EEG, and invasive electrophysiological data. Comput Intell Neurosci 2011:156869. CrossRef Medline

Oosterhof NN, Tipper SP, Downing PE (2013) Crossmodal and action-specific: neuroimaging the human mirror neuron system. Trends Cogn Sci 17:311-318. CrossRef Medline

Oosterhof NN, Connolly AC, Haxby JV (2016) CoSMoMVPA: multimodal multivariate pattern analysis of neuroimaging data in Matlab/ GNU Octave. Front Neuroinform 10:27. CrossRef Medline

Pineda JA (2005) The functional significance of mu rhythms: translating "seeing" and "hearing" into "doing." Brain Res Rev 50:57-68. CrossRef Medline

Pineda JA, Hecht E (2009) Mirroring and mu rhythm involvement in social cognition: are there dissociable subcomponents of theory of mind? Biol Psychol 80:306-314. CrossRef Medline

Pineda JO, Oberman LM (2006) What goads cigarette smokers to smoke? Neural adaptation and the mirror neuron system. Brain Res 1121:128135. CrossRef Medline

Ritchie JB, Tovar DA, Carlson TA (2015) Emerging object representations in the visual system predict reaction times for categorization. PLoS Comput Biol 11:e1004316. CrossRef Medline

Ritter P, Moosmann M, Villringer A (2009) Rolandic alpha and beta EEG rhythms' strengths are inversely related to fMRI-BOLD signal in primary somatosensory and motor cortex. Hum Brain Mapp 30:1168-1187. CrossRef Medline

Rossi S, Tecchio F, Pasqualetti P, Ulivelli M, Pizzella V, Romani GL, Passero S, Battistini N, Rossini PM (2002) Somatosensory processing during movement observation in humans. Clin Neurophysiol 113:16-24. CrossRef Medline

Singh F, Pineda J, Cadenhead KS (2011) Association of impaired EEG mu wave suppression, negative symptoms and social functioning in biological motion processing in first episode of psychosis. Schizophr Res 130:182186. CrossRef Medline

Tucciarelli R, Turella L, Oosterhof NN, Weisz N, Lingnau A (2015) MEG multivariate analysis reveals early abstract action representations in the lateral occipitotemporal cortex. J Neurosci 35:16034-16045. CrossRef Medline

Turella L, Tucciarelli R, Oosterhof NN, Weisz N, Rumiati R, Lingnau A (2016) Beta band modulations underlie action representations for movement planning. Neuroimage 136:197-207. CrossRef Medline

Vanderwert RE, Fox NA, Ferrari PF (2013) The mirror mechanism and mu rhythm in social development. Neurosci Lett 540:15-20. CrossRef Medline

Yang CY, Decety J, Lee S, Chen C, Cheng Y (2009) Gender differences in the mu rhythm during empathy for pain: an electroencephalographic study. Brain Res 1251:176-184. CrossRef Medline 